Meinung notwendig, seine Bedeutung für die atomistische Theorie, die heute noch keineswegs klar gestellt ist, einstweilen ganz aus dem Spiele zu lassen; und dieser Standpunkt entspricht gerade der auch sonst überall in diesem Buche befolgten Methode. Andrerseits habe ich dem Theorem, um seine Anwendungen so einfach wie umfangreich $\mathrm{zu}$ gestalten, eine möglichst weitgehende Fassung geben zu sollen geglaubt, und bin dabei, nicht nur in der Form, sondern auch inhaltlich, über die von NERNST selber gegebene noch etwas hinausgegangen. Ich erwähne diesen Punkt auch an dieser Stelle, weil die Möglichkeit im Auge zu behalten ist, dak, wenn die weitergehende Fassung sich nicht bewähren sollte, die ursprüngliche Nernstsche deswegen doch möglicherweise zu Recht bestehen bleiben könnte.

Berlin, im November 1910.

\title{
Vorwort zur sechsten und siebenten Auflage.
}

Unter den Ergänzungen, welche die neue Auflage erfahren hat, erwähne ich hier die von J. Св. GHоSH (Calcutta) aufgestellte Theorie für die Gefrierpunktsdepression starker Elektrolyte, welche die durch lange Zeit hindurch rätselhaft gebliebenen Abweichungen vom Ostwald schen Verdünnungsgesetz endgültig aufzuklären scheint ( $\$ 273)$, sowie die DeBYEsche Zustandsgleichung fester Körper, welche sowohl die Temperaturabhängigkeit der spezifischen Wärme als auch den GRÜNEISENschen Satz für den thermischen Ausdehnungskoeffizienten in sich enthält (§ 285).

Berlin-Grunewald, im Februar 1921 und im Juli 1922. 